\title{
Determinantes de la intención del consumidor de ir a un destino turístico. Aplicación al caso de "Mundo Maya-México"
}

\author{
Determinants of consumer intention to go to a \\ tourist destination. The case of "Mundo Maya- \\ México"
}

\author{
Dra. Sonia San Martín Gutiérrez ${ }^{1}$ \\ Rafael Fabricio Matos Cámara \\ Universidad de Burgos
}

Recibido el 8 de octubre de 2009, aceptado el 30 de septiembre de 2010

$\mathrm{N}^{\mathrm{o}}$ de clasificación JEL: M31

DOI: $10.5295 / \mathrm{cdg} .100178 \mathrm{ss}$

Reseña bibliográfica: SAN MARTÍN, S. y MATOS, R. F. (2011): "Determinantes de la intención del consumidor de ir a un destino turístico. Aplicación al caso de "Mundo Maya-México", Cuadernos de Gestión, Vol 11, n 1, pp.75-93, DOI: 10.5295/cdg.100178ss

\section{Resumen:}

Cada destino turístico tiene una marca que puede elegir el consumidor a la hora de viajar. Este trabajo examina, a través de un modelo causal que contrastamos empíricamente para el caso de Mundo Maya-México, el papel que desempeña la reputación, las emociones y la confianza en la intención de compra de los consumidores de un destino turístico. Desarrollamos un marco teórico fundamentado en aportaciones de la teoría de señales, las teorías de las emociones y del marketing de relaciones. Se ha realizado una encuesta personal a consumidores que acuden a las agencias de viajes. Los resultados obtenidos corroboran la mayoría de nuestras hipótesis y arrojan resultados interesantes: tanto la reputación de una buena gestión y alta notoriedad del destino como el agrado y la confianza del consumidor respecto del destino resultan determinantes de la intención del turista de ir a un destino turístico.

Palabras clave:

Intención de compra / reputación / emociones / confianza / destino turístico.

Abstract:

Each tourist destination has a brand the consumer can choose at the moment of travelling. This work analyzes, through a causal model empirically tested for the case of Mundo Maya-México, the paper that reputation, emotions and trust play in the consumer's intention to go to a tourist destination. A theoretical frame is developed using contributions of the signalling theory, emotions theories and relationship marketing. A face-to-face survey

\footnotetext{
${ }^{1}$ Departamento de Economía y Administración de Empresas. Facultad de Ciencias Económicas y Empresariales. Universidad de Burgos. C/ Parralillos, s/n, 09001 Burgos. E-mail: sanmargu@ubu.es.
} 
was carried out with consumers in travel agencies. Results confirm most of the proposed hypotheses and show interesting results: a reputation of a good management and high awareness of the tourist destination and consumer pleasure and trust in the destination reveal as antecedents of consumer intention to go to a certain tourist destination.

\section{Key Words:}

Intention of purchase / reputation / emotions / trust / tourist destination. 


\section{INTRODUCCIÓN}

En la actualidad la industria turística se encuentra en un cambio constante. En este sentido, los efectos de la globalización en los mercados han provocado grandes cambios en el nivel de competencia de los destinos turísticos. De tal modo, los destinos turísticos compiten por lograr despertar interés en el turista potencial y posicionarse en el lugar seleccionado al momento de su viaje. Ante esta situación, el enfoque de marketing tiene que ser otro y pasar de establecer simples acciones aisladas, que poco pueden contribuir por sí solas a la comercialización de la oferta turística de un destino, a una orientación hacia el mercado, en la que un enfoque hacia el turista y la competencia son los factores claves y a una orientación relacional que considere el papel de todos los stakeholders que se relacionan con un destino turístico (Morgan y Hunt, 1994; Acerenza, 2003).

Son muchos los trabajos de investigación sobre marketing que en las dos últimas décadas han tomado como referencia el marketing de relaciones (Ganesan, 1994; Morgan y Hunt, 1994; Gundlach et al., 1995; Doney y Cannon,1997; Ganesan y Hess, 1997; Wetzels et al.,1998; Garbarino y Johnson, 1999; Hess y Story, 2005) pero son menos los trabajos de marketing que contemplan postulados de la teoría de señales (Selnes, 1998; Erdem,1998; Kirmani y Rao, 2000) y de otras teorías, como las de emociones (Liljander y Strandvik, 1997; Bagozzi et al., 1999; Wong, 2004), más utilizadas en otras disciplinas, como la psicología. Los investigadores se han centrado en el análisis de algunos de estos enfoques y teorías tratándolos de forma particular, limitándose, casi siempre, a analizar el efecto por separado de cada uno de ellos. Este trabajo lo hemos abordado desde una variedad de aspectos como creemos que corresponde para entender un fenómeno como el turístico y así contemplamos aspectos económicos (teoría de la agencia y teoría de señales), aspectos relativos a la confianza (marketing relacional), y aspectos psicológicos (teoría de emociones).

Precisamente siguiendo la teoria de la agencia, podemos explicar una posible situación de información asimétrica entre los agentes (el consumidor y el destino turístico en nuestro caso), que se produce precisamente cuando el consumidor tiene que seleccionar la empresa más adecuada con la que desarrollar el intercambio o relación y no conoce la verdadera calidad de los productos que ofrece el vendedor o el verdadero comportamiento del mismo (Kirmani y Rao, 2000; Shapiro, 1982), en nuestro caso, el turista no conocería la calidad del destino turístico al que podría acudir. En primer lugar y respecto a la teoría de señales, los consumidores recurren a las marcas para inferir las características de los productos, debido a la existencia de información incompleta y asimétrica en los mercados. De tal modo, el consumidor toma decisiones basadas en la información de la cual dispone, y a partir de señales transmitidas por el vendedor (Kirmani y Rao, 2000). La reputación de marca (Kreps y Wilson, 1982; Erdem y Swait, 1998) es uno de los elementos clave utilizado para evaluar y elegir entre los distintos destinos turísticos, ante la variedad de productos existentes en el mercado. En segundo lugar, para incorporar a la investigación en marketing los efectos de las emociones, hemos revisado diversas teorías (Russell, 1980; Richins, 1997). En este trabajo valoramos las emociones del consumidor que le inspira un destino turístico previo a la decisión de elegir el destino al cual ir. De los enfoques que tratan el papel de las emociones en el comportamiento de compra, nos centramos especialmente en los que tratan los efectos de la anticipación de emociones (Bagozzi et al., 2003: 38). En tercer lugar, incluiremos algunas variables clave en el enfoque de marketing de relaciones que nos 
pueden ayudar a estudiar de una forma integral el comportamiento del turista. Zeithaml et al. (1996) enfatizaron la importancia de medir las intenciones del comportamiento futuro de los consumidores para determinar su lealtad. A pesar de la escasez de estudios que analicen la relación confianza e intención de compra hacia un destino turístico, la relación entre estas dos variables puede ser asimilada a la contrastada en otros ámbitos que nos proporciona la literatura siguiendo la teoría compromiso-confianza (Achrol, 1991; Morgan y Hunt, 1994; Macintosh y Lockshin, 1997; Farrelly y Quester, 2003).

Con la ayuda de este marco teórico, el objetivo es analizar el papel de la reputación del destino turístico, las emociones y la confianza del turista potencial como determinantes de la intención de ir a un destino. Trataremos el caso de los consumidores que tienen la intención de viajar, con información recogida de una muestra de castellano-leoneses. Este trabajo lo contrastaremos para el caso del destino turístico "Mundo-Maya-México", destino poco estudiado y de creciente interés en España y otras partes del mundo. Se les preguntará sobre esa región sureste de México a consumidores que no han estado allí. Las aportaciones de este trabajo a la literatura existente provienen de estudiar el binomio consumidordestino turístico utilizando un marco teórico derivado de la teoría de señales, las teorías de las emociones y el marketing de relaciones. Este trabajo resulta además importante por ser el primero aplicado al país y región objeto de estudio (Mundo Maya-México) y con la metodología de análisis path. Es más, se estudian relaciones entre variables como las emociones del turista y la reputación percibida del destino turístico o entre las emociones y la confianza, raramente estudiadas en el ámbito turístico.

\section{MARCO TEÓRICO}

\subsection{Teoría de señales y reputación de marca}

En una situación de selección adversa como la descrita previamente, en la que las empresas del destino turístico tienen información privada que el turista desconoce, podemos adoptar dos puntos de vista. Por un lado, el turista desea seleccionar aquel destino que sea más adecuado al que acudir, con empresas gestoras sin intenciones oportunistas y con tendencia al comportamiento cooperativo. La teoría de señales, enmarcada en la economía de la información, asume desde una perspectiva normativa que la información entre los distintos agentes económicos es imperfecta y asimétrica. Las señales en marketing son una actividad comercial que proporciona la información más allá de la actividad en sí misma. Estas señales se pueden enviar a los competidores, a los clientes, a los proveedores o a otros stakeholders (Herbig y Milewicz, 1996). El consumidor toma decisiones basadas únicamente en la información de la cual dispone, y a partir de las señales transmitidas por el vendedor, siendo el vendedor quien además tiene el poder para decidir la información que suministra (Kirmani y Rao, 2000). La información asimétrica entre las partes de una transacción como aquélla que hace que los consumidores especulen sobre información que desconocen de los productos o servicios de las empresas y realicen conclusiones a través de diversos atributos suministrados por los emisores, que se conocen como señales. La teoría de señales explica que los consumidores recurren a las señales para inferir las características de los productos, debido a la existencia de información incompleta y asimétrica en los mercados. 
De esta forma, el turista puede realizar esfuerzos de screening o selección de la mejor opción, mediante la recogida de información sobre las posibles alternativas de intercambio o relación y sobre las verdaderas características del destino y los gestores del mismo. Las señales que envían las empresas (agentes) al mercado ayudan en ese esfuerzo de búsqueda y selección por parte del consumidor. Las señales son las acciones que realiza la empresa para revelar de forma creíble su verdadero nivel de capacidades y la calidad no observable del producto, de una forma que no puedan imitar los proveedores de baja calidad (Kirmani y Rao, 2000; Singh y Sirdeshmukh, 2000; San Martín, 2003). Por otro lado, las empresas gestoras del destino pueden estar interesadas en indicar que su destino es una buena opción para el establecimiento de un intercambio, para un viaje, y mostrarse a sí mismas como empresas que gestionan y ofrecen un destino de calidad para que el turista lo elija para su viaje. En este caso, diríamos que el destino trata de autoseleccionarse como el que mejor se ajusta a las condiciones requeridas por los turistas potenciales (Bergen et al., 1992). Para ello, la empresa utiliza señales para favorecer el establecimiento del intercambio con él/ella.

Para los fines de este estudio, trataremos la reputación de marca como una señal del destino turístico (Kreps y Wilson, 1982; Chu y Chu, 1994; Erdem y Swait, 1998). La reputación de marca es uno de los elementos utilizados para evaluar y elegir entre los distintos destinos turísticos, ante la variedad de productos turísticos existentes en el mercado. La reputación da al consumidor información, permitiéndole evaluar las distintas alternativas y de este modo poder tomar una decisión de compra satisfactoria en su intención de ir al destino turístico. La reputación del destino turístico resume así la información sobre la empresa y es utilizada por el consumidor para reducir el esfuerzo de búsqueda de información y el riesgo percibido en la elección del lugar (Memelsdorff, 1998; Erdem y Swait, 1998). Siguiendo la revisión de señales realizada por Kirmani y Rao (2000), la inversión en reputación es una señal que puede conllevar una pérdida de imagen de la empresa si ésta indica una calidad falsa. La empresa que invierte en formar una reputación arriesga sus ingresos futuros e incluso su propia supervivencia (Herbig y Milewicz, 1995) si los clientes perciben que la reputación conseguida a lo largo del tiempo ya no corresponde con el comportamiento de la empresa o con la calidad de sus productos (Shapiro, 1982).

\subsection{Teorías y efectos de las emociones en marketing}

Las emociones son un área de investigación importante en el campo del marketing en los últimos años. En la literatura se ha demostrado que las emociones tienen una influencia en el comportamiento y responden a acontecimientos que en cierto modo implican mantener emociones positivas (e.g. felicidad) y evitar emociones negativas (e.g. tristeza) (Stauss y Neuhaus, 1997; Bagozzi, 1997). Así, es necesario acudir a disciplinas como la psicología para estudiar las emociones en marketing y tener una visión integral en la comprensión y entendimiento del consumidor (Izard, 1977, Plutchik,1980; Watson y Clark, 1992; Huang, 2001).

Mientras Russell (1980), Plutchnik (1980), Frijda (1986) se pueden considerar enfoques que analizan los efectos de las emociones experimentadas en el presente, otros como Bagozzi, Wilson o Parker et al. (ver revisión en Bagozzi et al., 2003) se centran en los efectos comportamentales de la anticipación de emociones. 
Para su aplicación al marketing, habría que contemplar las características de las emociones que intervienen de forma específica en el comportamiento del consumidor (Huang, 2001). Aunque no parece haber una definición de común acuerdo sobre esta variable entre los investigadores de marketing, nosotros tratamos la variable emociones siguiendo a Russell (1980), como una apreciación subjetiva de lo agradable o desagradable, haciendo referencia al nivel en el cual una persona muestra un afecto positivo o negativo (e.g. feliz o infeliz) en una determinada situación.

\subsection{Marketing de relaciones y confianza}

A lo largo de la última década, numerosos autores han llegado a la conclusión de que el elemento central en marketing son las relaciones. Estas relaciones son contactos entre dos o más personas, pero también se dan entre personas y objetos, símbolos y organizaciones (Gummesson, 1996). En las investigaciones de marketing de relaciones no se deben analizar tan sólo los intercambios entre compradores y vendedores como situaciones simples o transacciones discretas, sino como relaciones continuas en el tiempo. Es por ello que surge la necesidad de realizar un esfuerzo integrado por mantener y consolidar el intercambio con los clientes a través del tiempo.

El compromiso y la confianza son dos elementos fundamentales en los intercambios y relaciones como refleja la teoría compromiso-confianza desarrollada por Morgan y Hunt (1994). Precisamente esta teoría trata de hacer frente a una de las críticas que se han realizado al marketing de relaciones: la carencia de una teoría que otorgue contenido al enfoque. En su trabajo, donde presentan el modelo key mediating variable, comúnmente conocido como modelo compromiso-confianza, se incluyen por primera vez de forma explícita el compromiso y la confianza como variables clave en las relaciones.

Así algunos autores sostienen la postura de que la confianza es un factor determinante para conseguir el compromiso en una relación (Achrol, 1991; Morgan y Hunt, 1994; Gundlach et al., 1995; San Martín, 2003) y que es una variable clave para ayudar al consumidor a discernir en sus decisiones (Selnes, 1998; Hess y Story, 2005). En el ámbito que nos ocupa, el turístico, es evidente la importancia de atraer turistas y generar confianza y compromiso para mantener relaciones duraderas con los turistas y que tanto empresas como clientes se beneficien del valor obtenido de esas relaciones de fidelidad (ejemplo efecto boca-oído). No obstante, dada la búsqueda de variedad de destinos por parte del consumidor, es difícil para los gestores de los destinos conseguir la repetición de compra del consumidor y, por tanto, su compromiso.

En la literatura relacional, la confianza se ha tratado de muy diversas maneras, pero casi siempre de una forma parcial e incompleta. Así, la confianza ha sido definida como un deseo de fiarse de otra parte (Andaleeb, 1996), una esperanza o expectativa de que la otra parte colaborará en la relación (Dwyer, et al., 1987), una norma social que mitiga el riesgo de oportunismo (Sako y Helper, 1998), una creencia en la posibilidad de que el otro coopere (Nooteboom y Noorderhaven, 1997) o un estado psicológico que se concreta en la intención de aceptar una vulnerabilidad al comportamiento del otro (Singh y Sirdeshmukh, 2000). Tomando en consideración todas las acepciones anteriores, entendemos por confianza la seguridad emocional de una parte que le lleva a pensar que la otra parte 
es responsable y se preocupará por ella, lo cual implica la disposición de la primera a ser vulnerable a las acciones de la segunda, independientemente de la capacidad de aquélla de controlar a esta última (Moorman et al., 1993).

\section{DESARROLLO DE LAS HIPÓTESIS Y MODELO PROPUESTOS}

\subsection{Relación entre las emociones y la reputación percibida del destino}

En la actualidad, gran cantidad de destinos turísticos basan su posicionamiento en beneficios emocionales. Estas emociones pueden reflejarse en felicidad o alegría cuando el individuo piensa en un determinado entorno (Restall y Gordon, 1993). Al igual que las relaciones que tenemos con personas en situaciones sociales u objetos están conducidas por nuestras emociones, la relación del consumidor con un destino turístico al que viajar se torna como una relación emocional (Gobé, 2001; Hess y Story, 2005). Siguiendo a Gardner (1985), podemos proponer que las emociones que anticipan los consumidores sobre una empresa influyen en sus evaluaciones del producto y percepciones sobre la empresa, confirmándose mayores evaluaciones positivas cuando el consumidor muestra unas emociones positivas (Clark e 1sen, 1982; Bagozzi et al., 2003: 37-38). Así, proponemos que las emociones que un destino turístico inspira a un consumidor influirán en su percepción de la reputación del destino turístico.

H1: Las emociones positivas que experimenta el consumidor hacia el destino turístico influyen positivamente en la reputación percibida de ese destino.

\subsection{Determinantes de la confianza en el destino turístico}

En las relaciones empresa-consumidor, el desconocimiento de información relevante sobre la empresa y la imposibilidad de controlar directa y totalmente su comportamiento hacen que el consumidor tenga que asumir un riesgo al oportunismo de la empresa, un riesgo que se puede mitigar mediante señales como la reputación. De esta forma, la literatura ha considerado la reputación como un factor dominante para crear confianza entre las organizaciones y los consumidores en sus intercambios (Ganesan, 1994, Doney y Cannon, 1997) y en el contexto de la comercialización de los destinos turísticos (Memelsdorff, 1998). Mitchell y Vincent-Wayne (1999) sugieren que la reputación afecta a la interpretación actitudinal de la calidad y genera confianza. En esta misma línea, Moorman et al. (1993) contemplan la reputación como un indicador de la confiabilidad. Por lo tanto, desde este punto de vista, es de esperar que una reputación buena y fuerte reduzca el riesgo de compra de los clientes potenciales y de este modo genere confianza (Autores, 2009). De acuerdo con el argumento anterior:

H2: La reputación percibida de un destino turístico influye positivamente en la confianza del consumidor en ese destino.

En el marketing aplicado a un destino turístico, también resulta trascendente estudiar la interacción entre las emociones y la confianza. El marketing desarrolla y explica las 
emociones como factores influyentes en su comportamiento (Oliver, 1997; Derbaix y Phan, 1998; Bagozzi et al., 1999). De hecho, entre los efectos de la anticipación de las emociones podemos señalar reacciones afectivas como las que subyacen en la confianza (Bagozzi et al., 2003: 38). La seguridad implícita en la confianza será mayor cuanto más positivas sean las emociones que el destino turístico despierta en el consumidor (Dunn y Schweitzer, 2005). Esta investigación pretende confirmar esta relación para los destinos turísticos:

H3: Las emociones positivas que siente el consumidor hacia un destino turístico influyen positivamente en la confianza del consumidor con ese destino.

\subsection{Determinantes de la intención de ir al destino turístico}

La confianza y el compromiso han sido relacionados en distintos trabajos y contextos siguiendo la teoría compromiso-confianza del marketing relacional (Anderson y Weitz, 1992; Ganesan, 1994; Gundlach et al., 1995; Morgan y Hunt, 1994; Garbarino y Johnson, 1999). Sin embargo, hay menos estudios que analicen la relación entre confianza e intención de compra (Macintosh y Lockshin, 1997) y menos aún aplicados a la intención de ir a un destino turístico. El efecto positivo de la confianza sobre la repetición de comprar y el compromiso parece estar relacionado con el valor que genera en el individuo la mayor confiabilidad percibida en la otra parte (Morgan y Hunt, 1994; Ganesan y Hess, 1997; Doney y Canon, 1997; Wetzels et al., 1998; Garbarino y Johnson, 1999). Zeithaml et al., (1996) enfatizaron la importancia de medir las intenciones del comportamiento futuro de los consumidores para determinar su potencial de permanecer o de abandonar la organización. Quienes muestran unas actitudes favorables hacia la organización manifiestan una intención de compra mayor (Bellman et al., 1999). De acuerdo con los razonamientos precedentes y centrándonos en la intención de ir a un destino turístico:

H4: La confianza del consumidor en un destino turístico influye positivamente en la intención del consumidor de ir a ese destino.

Las emociones regulan e influyen en nuestro comportamiento e incluso lo organizan (Oliver, 1997; Derbaix y Phan, 1998; Bagozzi et al., 1999). Las emociones generan nuestros deseos y permiten entablar asociaciones con el destino turístico. Por ello, los consumidores eligen el destino turístico esperando que éste le ofrezca un conjunto de beneficios a largo plazo de acuerdo con las emociones que le inspira ese lugar. Siguiendo a Bagozzi et al. (2003: 37), la anticipación de futuras experiencias emocionales donde el individuo simula mentalmente eventos en los que las emociones son relevantes, como es el caso turístico, tienen efectos importantes en el comportamiento. Basándonos en Russell (1980), proponemos un efecto positivo de las emociones en la intención de compra (intención de ir) hacia el destino turístico. Es de esperar que quienes muestren emociones positivas respecto de la organización manifiesten una mayor intención de compra (Bellman et al., 1999; Goosens, 2000). Por tanto:

H5: Las emociones positivas que siente el consumidor hacia un destino turístico influyen positivamente en la intención del consumidor de ir a ese destino. 


\section{METODOLOGÍA}

En nuestra investigación se ha optado por analizar la relación entre el consumidor y un destino turístico. Se decidió elegir la relación que involucrara al consumidor final y en la cual se reflejaran conceptos como la reputación de marca, las emociones, la confianza, y la intención de compra (intención de ir). Por esa razón se eligió un producto de gran complejidad de compra debido a sus características intangibles como es un destino turístico. El producto intangible analizado, un destino turístico, tiene implicaciones emocionales, especialmente si el fin es el ocio o las vacaciones.

La investigación empírica se aplicó al destino turístico "Mundo Maya-México" (región sureste de México). Para el estudio empírico se realizaron encuestas personales dentro de las agencias de viajes de una ciudad de Castilla y León con el permiso del encargado en cada caso tras explicarles brevemente la investigación en proceso de realización en la Universidad. Se acudió a todas las agencias de viajes de la ciudad, aunque principalmente colaboraron las que ofrecen viajes dirigidas a jóvenes y grupos y se les preguntó a los consumidores dentro de las agencias, antes de contratar su viaje, sobre su actitud e intención de ir a Mundo Maya México (pero sin folletos ni imágenes y sin ningún tipo de información adicional). La ficha técnica del trabajo de campo aparece en la Tabla 1.

\section{Tabla 1}

Ficha Técnica del estudio y características de la muestra

\begin{tabular}{|c|c|}
\hline Características & Encuesta \\
\hline Universo & $\begin{array}{l}\text { - Personas que acuden a la agencia de viajes con intención de viajar por placer } \\
\text { (potenciales turistas españoles a México). }\end{array}$ \\
\hline Área geográfica & - Una gran ciudad de Castilla y León. \\
\hline Tamaño muestral & $\begin{array}{l}\text { - Se recibieron } 201 \text { encuestas válidas de un total de } 290 \text { encuestas entregadas } \\
\text { (tasa de respuesta }=69,3 \% \text { ). }\end{array}$ \\
\hline Diseño muestral & - La información fue recogida mediante encuesta personal. \\
\hline Error muestral & - $6.92 \%$ (para el caso más desfavorable: $\mathrm{p}=\mathrm{q}=0.5$ ). \\
\hline $\begin{array}{l}\text { Características } \\
\text { de la muestra }\end{array}$ & $\begin{array}{l}\text { - Sexo: } 43 \% \text { hombres y } 57 \% \text { mujeres. } \\
\text { - Estructura del hogar: el } 25 \% \text { viven de manera unipersonal, el } 18 \% \text { pareja sin } \\
\text { hijos, el } 50 \% \text { pareja con hijos y el } 7 \% \text { uno más hijos (sólo una persona de la } \\
\text { pareja más hijos). } \\
\text { - Edad: el } 4 \% \text { de los encuestados tienen menos de } 20 \text { años, el } 50 \% \text { entre } 21 \text { y } \\
30 \text { años, el } 28 \% \text { entre } 31 \text { y } 40 \text { años, el } 10 \% \text { entre } 41 \text { y } 50 \text { años, el } 5 \% \text { entre } \\
51 \text { y } 60 \text { años y el } 3 \% \text { más de } 60 \text { años. } \\
\text { - Nivel de estudios: } 0.5 \% \text { de la muestra carece de estudios, } 1 \% \text { posee estudios } \\
\text { primarios, } 23 \% \text { posee estudios medios y } 75.5 \% \text { posee estudios superiores. } \\
\text { - Profesión: el } 34 \% \text { es trabajador por cuenta ajena, el } 1.5 \% \text { empresarios, el } 5 \% \\
\text { autónomo, el } 2 \% \text { jubilado, el } 20 \% \text { funcionario, el } 30.5 \% \text { estudiante, el } 2 \% \\
\text { ama de casa y el } 5 \% \text { desempleado. } \\
\text { - Renta mensual neta del hogar: el } 16.4 \% \text { perciben menos de } 600 € \text {; el } 11.1 \% \\
\text { entre } 601-900 € \text {; el } 16.4 \% \text { entre } 901-1200 € \text {; el } 16.4 \% \text { entre } 1201-1500 € \text {; } \\
\text { el } 6.5 \% \text { entre } 1501-1800 € \text {; el } 16.4 \% \text { entre } 1801-2400 € \text {; el } 10.3 \% \text { entre } \\
2401-3000 € \text {; el } 6.5 \% \text { más de } 3000 € \text {. }\end{array}$ \\
\hline
\end{tabular}


Con la muestra, hemos observado que los estados de "Mundo Maya-México" más conocidos por los españoles son: Quintana Roo con 69\%, Yucatán 58\%, y Chiapas $46 \%$ y los menos conocidos: Tabasco $12.5 \%$ y Campeche $8.5 \%$, éste ultimo estado se sitúa como el que menos frecuencia de conocimiento presenta en los turistas de la muestra. Aunque todos estos lugares crean el destino en su totalidad, sólo el $41 \%$ de las personas de la muestra manifestaron su conocimiento de Mundo Maya-México, porque no lo relacionan con los lugares que integran este destino turístico. Una razón de estos resultados puede ser la mayor promoción y publicidad de determinados lugares como Cancún y Riviera Maya (ambos destinos pertenecientes a Quintana Roo) en España que puede hacer que muchos turistas lo confundan con Mundo Maya-México. ${ }^{2}$

Para diseñar las escalas que nos permitiesen cuantificar las variables de reputación, emociones, confianza, intención de compra (intención de ir) hacia el destino turístico "Mundo Maya-México", se tomaron como punto de partida las principales escalas desarrolladas en la literatura sobre estas variables. De manera concreta se consideró a Fombrun (1998) y Bennet y Gabriel (2001) para la escala de reputación. Las emociones fueron medidas a partir de la escala de Russell (1980) y Huang (2001). Para la escala de confianza se consideraron especialmente los trabajos de Ganesan (1994), Evans y Laskin (1994), Macintosh y Lockshin (1997) y Bart et al. (2005). Por otra parte, para la escala en la intención de compra (intención de ir) se consideraron las escalas de Macintosh y Lockshin (1997) y Garbarino y Johnson (1999). Todas las variables fueron medidas a través de escalas tipo Likert de 5 posiciones (desde totalmente en desacuerdo a totalmente de acuerdo con la proposición formulada), excepto la de las emociones (escala de diferencial semántico de cinco posiciones) y las variables sexo, estructura del hogar, edad, nivel de estudios, profesión y renta mensual neta del hogar. Las variables principales y sus ítemes aparecen codificados y descritos en el Apéndice.

\section{RESULTADOS DEL ESTUDIO EMPÍRICO}

Tras realizar un análisis univariante y bivariante, y verificar que no existían anomalías significativas en los datos, se procedió a depurar las escalas iniciales a través de un análisis factorial exploratorio (análisis de ejes principales con rotación varimax), el cual nos permitió elegir los ítemes que tienen una carga factorial alta (Hair et al., 1999). En el análisis factorial realizado, se observa la existencia de tantos factores como variables habíamos considerado excepto para el caso de la reputación, que engloba a dos factores que denominamos reputación de gestión y reputación de notoriedad. El factor de reputación de gestión se relaciona con la buena gestión, la calidad ofrecida de productos y servicios turísticos, la responsabilidad del medio ambiente y la utilización inteligente de estos recursos en el destino turístico, mientras que la reputación de notoriedad se refiere al conocimiento del destino en los medios de comunicación, la familiaridad con destinos turísticos similares

\footnotetext{
${ }^{2}$ Es importante mencionar que pese a que en los últimos años muchos españoles han acudido a México por motivos turísticos y como se observa en este estudio, existen muy pocas personas que conocen los lugares de Mundo Maya-México. El destino turístico “Mundo Maya-México” está integrado por cinco estados del sureste mexicano: Campeche, Chiapas, Quintana Roo, Tabasco y Yucatán. Así, Cancún y Riviera Maya (dos de los destinos más visitados por los turistas españoles) pertenecen ambos al estado de Quintana Roo.
} 
a éste y a la buena reputación en sí misma. En la literatura otros trabajos también hacen mención a diferentes dimensiones de la reputación (Resnick, 2003). Pensando si las dos dimensiones pueden estar relacionadas, parece lógica pensar que el hecho de que un lugar turístico esté bien gestionado a través del uso racional de sus recursos, de la calidad que se ofrece, y de lo ambientalmente responsable que éste sea, influirá positivamente en la percepción de los consumidores, a través de una buena reputación de notoriedad, de que los medios de comunicación mencionan el lugar con frecuencia y en su familiaridad con el lugar. Por ello, relacionamos esas dos dimensiones claramente obtenidas en el análisis factorial en la siguiente hipótesis:

H6: La reputación de gestión percibida del destino turístico influye positivamente en la reputación de notoriedad percibida de ese destino.

Tras realizar el análisis factorial exploratorio para el conjunto de variables, hemos hecho un análisis factorial confirmatorio para todas ellas con el objetivo de comprobar la validez convergente y discriminante de las escalas y depurar éstas para simplificarlas, hasta llegar a las definitivas con la ayuda del programa estadístico LISREL 8.7. Los resultados del análisis factorial confirmatorio (tras haber eliminado oportunamente determinadas variables del modelo, V11, V12, V14 y V20) aparecen en la Tabla 2. En esta tabla también se muestran los valores del coeficiente alpha de Cronbach, de la fiabilidad compuesta y de la varianza extraída y en todos los casos los valores exceden los recomendados de 0.70, 0.60 y 0.50 respectivamente (Fornell y Larcker, 1981). Si valoramos la parsimonia del modelo, observamos que el criterio de información de Akaike (AIC) y el consistente (CAIC) son inferiores en el modelo estimado que en el modelo saturado y en el modelo de variables independientes, lo que indica parsimonia del modelo (Hair et al., 1999).

Tabla 2

\section{Resultados del análisis factorial confirmatorio}

\begin{tabular}{|c|c|c|c|c|c|c|c|c|}
\hline \multirow{2}{*}{$\begin{array}{l}\text { Variable } \\
\text { latente }\end{array}$} & \multirow{2}{*}{\multicolumn{2}{|c|}{ Variable de medida }} & \multicolumn{2}{|c|}{ Coeficientes lambda } & \multirow{2}{*}{$\mathrm{R}^{2}$} & \multirow{2}{*}{$\begin{array}{l}\text { Alpha de } \\
\text { cronbach }\end{array}$} & \multirow{2}{*}{$\begin{array}{l}\text { Fiabilidad } \\
\text { compuesta }\end{array}$} & \multirow{2}{*}{$\begin{array}{l}\text { Varianze } \\
\text { extraída }\end{array}$} \\
\hline & & & Lambda & $t$ & & & & \\
\hline \multirow{4}{*}{$\begin{array}{l}\text { Reputación } \\
\text { de gestión }\end{array}$} & $V 9$ & Creencia en buena gestión & 1.000 & -- & 0.644 & \multirow{4}{*}{0.71} & \multirow{4}{*}{0.812} & \multirow{4}{*}{0.68} \\
\hline & V10 & Representa calidad & 1.012 & 16.094 & 0.725 & & & \\
\hline & $V 11$ & $\begin{array}{l}\text { Ambientalmente respon- } \\
\text { sable }\end{array}$ & \multicolumn{3}{|c|}{ Eliminada } & & & \\
\hline & V12 & $\begin{array}{l}\text { Utiliza recursos de forma } \\
\text { inteligente }\end{array}$ & \multicolumn{3}{|c|}{ Eiminada } & & & \\
\hline \multirow{3}{*}{$\begin{array}{l}\text { Reputación } \\
\text { de notorie- } \\
\text { dad }\end{array}$} & V13 & $\begin{array}{l}\text { Mención en medios de } \\
\text { comunicación }\end{array}$ & 1.000 & -- & 0.602 & \multirow{3}{*}{0.70} & \multirow{3}{*}{0.801} & \multirow{3}{*}{0.801} \\
\hline & V14 & $\begin{array}{l}\text { Familiarizado con lugares } \\
\text { similares }\end{array}$ & \multicolumn{3}{|c|}{ Eliminada } & & & \\
\hline & V15 & Buena reputación & 1.053 & 17.422 & 0.735 & & & \\
\hline
\end{tabular}


Determinantes de la intención del consumidor de ir a un destino turístico...

\begin{tabular}{|c|c|c|c|c|c|c|c|c|}
\hline \multirow{2}{*}{$\begin{array}{l}\text { Variable } \\
\text { latente }\end{array}$} & \multirow{2}{*}{\multicolumn{2}{|c|}{ Variable de medida }} & \multicolumn{2}{|c|}{ Coeficientes lambda } & \multirow{2}{*}{$\mathrm{R}^{2}$} & \multirow{2}{*}{$\begin{array}{l}\text { Alpha de } \\
\text { cronbach }\end{array}$} & \multirow{2}{*}{$\begin{array}{l}\text { Fiabilidad } \\
\text { compuesta }\end{array}$} & \multirow{2}{*}{$\begin{array}{l}\text { Varianza } \\
\text { extraída }\end{array}$} \\
\hline & & & Lambda & $t$ & & & & \\
\hline \multirow{6}{*}{ Emociones } & V16 & Feliz /infeliz & 0.860 & 15.077 & 0.612 & \multirow{6}{*}{0.90} & \multirow{6}{*}{0.919} & \multirow{6}{*}{0.69} \\
\hline & V17 & Contento/Enfadado & 1.032 & 21.146 & 0.818 & & & \\
\hline & V18 & Encantado/Descontento & 1.000 & - & 0.758 & & & \\
\hline & V19 & Alegre/Triste & 0.978 & 18.966 & 0.753 & & & \\
\hline & V20 & Ilusionado/Desilusionado & \multicolumn{3}{|c|}{ Eliminada } & & & \\
\hline & V21 & Entretenido/Aburrido & 0.896 & 16.145 & 0.654 & & & \\
\hline \multirow{5}{*}{ Confianza } & V22 & $\begin{array}{l}\text { Creencia cumple sus } \\
\text { promesas }\end{array}$ & 0.827 & 12.854 & 0.546 & \multirow{5}{*}{0.80} & \multirow{5}{*}{0.885} & \multirow{5}{*}{0.61} \\
\hline & V23 & $\begin{array}{l}\text { Creencia conoce a sus } \\
\text { clientes }\end{array}$ & 0.896 & 14.331 & 0.619 & & & \\
\hline & V24 & Información detallada & 0.789 & 12.073 & 0.506 & & & \\
\hline & V25 & $\begin{array}{l}\text { Satisfacción hacia el } \\
\text { cliente }\end{array}$ & 1.000 & - & 0.674 & & & \\
\hline & V26 & $\begin{array}{l}\text { Características a gustos y } \\
\text { preferencias }\end{array}$ & 0.766 & 12.122 & 0.473 & & & \\
\hline \multirow{2}{*}{$\begin{array}{l}\text { Intención } \\
\text { de compra } \\
\text { (intención } \\
\text { de ir) }\end{array}$} & V27 & Ir al lugar $\mathrm{X}$ en el futuro & 1.000 & - & 0.659 & \multirow[b]{2}{*}{0.69} & \multirow[b]{2}{*}{0.728} & \multirow[b]{2}{*}{0.60} \\
\hline & V28 & No descarto ir al lugar $\mathrm{X}$ & 0.817 & 11.227 & 0.539 & & & \\
\hline & 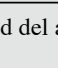 & $\begin{array}{l}\text { ste: } \chi^{2}(98)=230.888(\mathrm{p}=0 \\
\text { AIC modelo estimado }=2\end{array}$ & $\begin{array}{l}\text { MSEA } \\
5, \text { inde }\end{array}$ & nte $=2$ & 920 & $.952 \mathrm{II}$ & $.952 \mathrm{GF}$ & 90 \\
\hline
\end{tabular}

La matriz de correlaciones entre las variables latentes nos indica que las cinco variables están correlacionadas pero son variables diferentes. No obstante, para comprobar el poder discriminante entre las variables latentes, se estimó un modelo confirmatorio de las variables latentes en conjunto, fijando a 1 las correlaciones y la bondad del ajuste es peor que en el modelo sin restricciones (Tabla 3). Asimismo, se comprobó que la correlación al cuadrado entre las variables fuese inferior a la varianza extraída para cada una de ellas.

Tabla 3

Matriz de correlaciones entre las variables latentes

\begin{tabular}{|l|r|r|r|r|r|}
\hline & \multicolumn{1}{|c|}{ REPGES } & \multicolumn{1}{c|}{ REPNOT } & EMOCIONES & CONFIANZA & INTENCIÓN \\
\hline REPGES & 1 & & & & \\
\hline REPNOT & 0.706 & 1 & & & \\
\hline EMOCIONES & 0.361 & 0.528 & 1 & & \\
\hline CONFIANZA & 0.603 & 0.612 & 0.378 & 1 & \\
\hline INTENCIÓN & 0.255 & 0.324 & 0.469 & 0.336 & 1 \\
\hline
\end{tabular}


Para la estimación del modelo global, realizamos un análisis path, considerando los modelos de medida previamente estimados resultando del proceso de depuración de cada escala. Los índices de bondad del ajuste son buenos y la estimación muestra que se cumplen todas las hipótesis excepto H3 (Gráfico 1).

\section{Gráfico 1}

\section{Resultados de la estimación del modelo final}

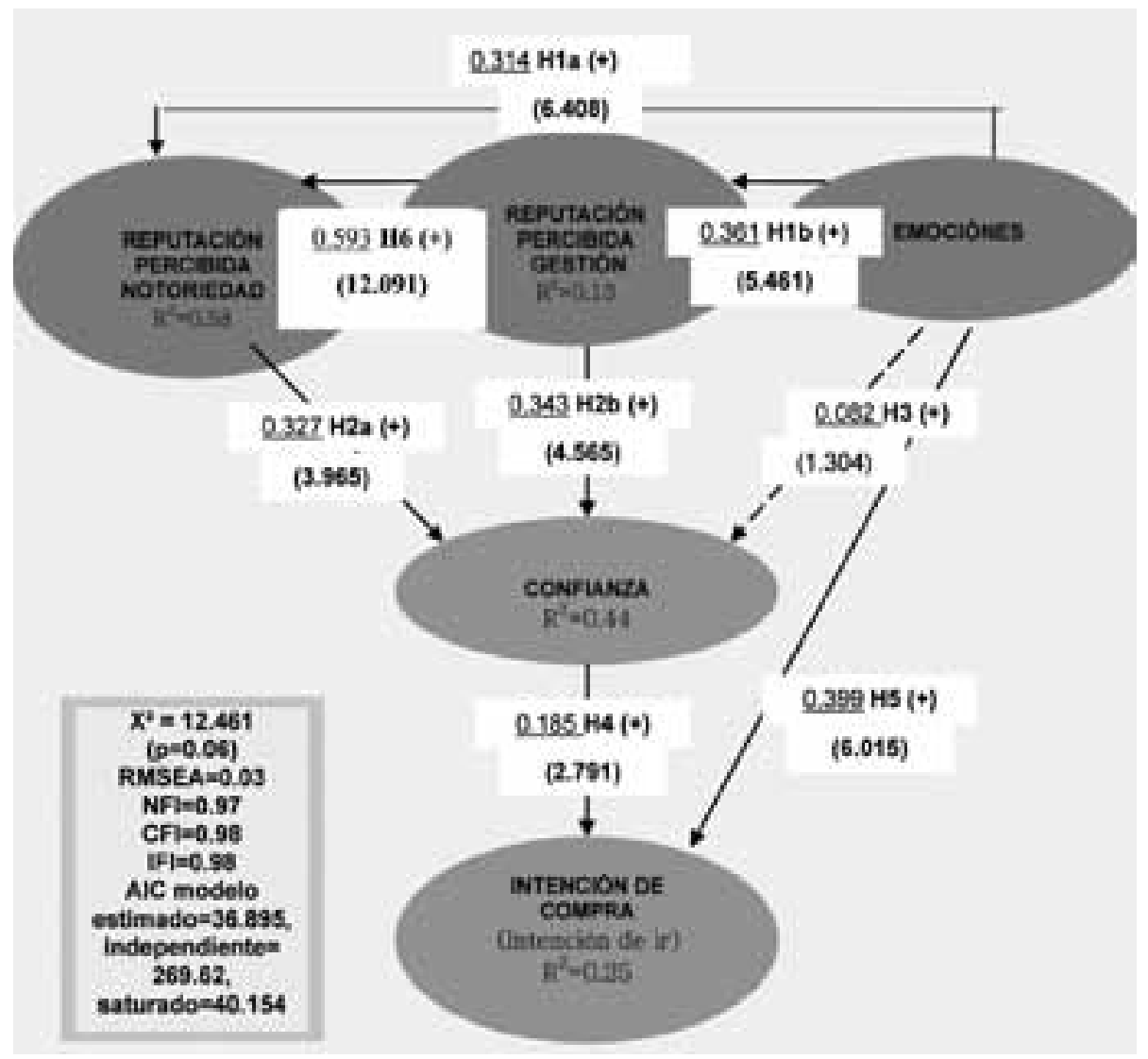

\section{CONCLUSIONES}

El estudio empírico, realizado a partir de la información suministrada por una muestra de usuarios de agencias de viajes con la intención de ir hacia un destino turístico, surge de la pretensión de conocer las interrelaciones entre la reputación del destino y la actitud, emociones e intención del turista de acudir a un destino. Hemos contrastado el modelo para el caso de Mundo Maya-México dada la repercusión que ha tenido este destino en los últimos años en España. Es importante analizar el hecho de la falta de información que se tiene 
del destino Mundo Maya-México, lo que aumenta el problema de selección adversa al que se enfrenta el turista y que hace necesario que los gestores del destino difundan éste en los medios de comunicación y lograr presencia en el mercado para contribuir al crecimiento económico de esta región.

Los resultados apoyan todas las hipótesis propuestas excepto una y arrojan implicaciones interesantes para los investigadores y profesionales de marketing. Tras haber encontrado dos tipos de reputación del destino turístico -la reputación de una buena gestión del destino y la reputación relacionada con una alta notoriedad del destino-, tratamos de conocer la relación entre ellas y encontramos que la reputación de gestión del destino influye positivamente en la reputación de notoriedad. Esto indica la necesidad de gestionar bien un destino turístico antes de comunicarlo porque el consumidor valora más esa buena gestión que la publicidad del destino. Por lo que se refiere al resto de hipótesis, observamos que tanto la reputación de gestión como la reputación de notoriedad percibidas respecto de un determinado destino turístico contribuyen a generar o aumentar la confianza en el destino. Y es que la reputación funciona como una señal de calidad del destino que reduce el problema de selección adversa del turista que se enfrenta a la decisión de elección del destino reduciendo el riesgo y aumentando la confianza. Así se corrobora para el caso de los destinos turísticos el papel de la reputación como señal generadora de confianza, relación ya encontrada en otros trabajos para otros contextos (Moorman et al., 1993; Ganesan, 1994; Kirmani y Rao, 2000; Autores, 2009). Y es que la reputación como recurso intangible aumenta el valor comercial de los destinos, a través de la creación de ventajas diferenciales en ambientes tan competitivos como el turístico.

Además, siguiendo los efectos de la anticipación de emociones que sugieren Bagozzi et al. (2003), podemos concluir que las emociones positivas que anticipa el turista respecto de un determinado destino inciden positivamente en su percepción de la reputación del destino, tanto en su vertiente de notoriedad como en la de gestión y con un efecto de tamaño similar. Este resultado corrobora que las emociones inciden en las percepciones respecto de la empresa o destino en nuestro caso (Clark e 1sen, 1982; Gardner, 1985). Por lo que se refiere al efecto de las emociones positivas del turista en la confianza e intención de acudir a un destino, hay que señalar que el primer efecto propuesto (efecto indirecto de las emociones sobre la intención a través de la confianza) no es significativo estadísticamente y el segundo sí (efecto directo de las emociones positivas en la intención de compra). Dunn y Schweitzer (2005) también encontraron que en determinadas circunstancias, las emociones no influyen en la confianza. Además, uno de los mayores efectos del modelo muestra que la anticipación de emociones positivas del consumidor hacia el destino turístico incide directamente en la intención de "compra" del destino. Este resultado indica la fuerza de esta variable a la hora de comprar, por lo que los gestores de un determinado destino deben apelar a emociones positivas como la felicidad o alegría que reporta ir a un determinado destino turístico y crear las condiciones propicias previas a la compra para comunicar y publicitar un destino y ser decisivos así en la intención de compra del turista. No obstante, esas emociones positivas previas pueden suponer mayores expectativas que después deben ser satisfechas cuando el turista acude a un determinado destino turístico. En futuros trabajos se podría tratar este punto.

Por último, un mayor grado de confianza hace que el consumidor tenga intención de ir al destino turístico, lo que confirma una vez los postulados de la teoría compromiso- 
confianza, al menos en su primera fase de confianza-intención de compra (Macintosh y Lockshin, 1997; Bellman et al., 1999). Posteriormente, si se sigue reforzando el marketing de relaciones, la intención de compra podría conducir a una repetición de compras, lealtad y prescripción de ese destino mediante el efecto boca-oreja.

\section{LIMITACIONES Y LÍNEAS FUTURAS DE INVESTIGACIÓN}

En cuanto a las limitaciones, podemos considerar el estrecho ámbito geográfico, la referencia del estudio hacia un solo producto (un destino turístico concreto) y la composición mayoritariamente universitaria de la muestra, que como consecuencia nos restringe las posibilidades de generalizar las conclusiones del trabajo. Asimismo, la naturaleza del tipo de estudio realizado, ya que es de corte transversal y no nos permite conocer empíricamente sin ambigüedad el sentido de las relaciones causales (por ejemplo, entre reputación y emociones), pese a que están justificadas teóricamente en el sentido propuesto. Otra limitación es la medición de la variable intención de compra/compromiso con sólo dos ítemes y los índices y coeficientes de bondad del ajuste son mejorables. En esta misma línea hemos estudiado las emociones que inspira al consumidor un destino turístico antes de viajar y no las emociones después del viaje de los consumidores que sería más adecuado. Finalmente hemos estudiado sólo la perspectiva del consumidor y dejar en este momento fuera del estudio las acciones que realiza el destino turístico. Por supuesto, hay que reconocer que si el estudio se realizase en otros momentos como el año pasado, afectado por aspectos como la gripe $\mathrm{A}$, los resultados podrían ser diferentes, pero es una circunstancia puntual.

Como líneas para futuras investigaciones, proponemos aumentar la recogida de información a nivel nacional y a otros países (Europa y Latinoamérica), para tener una visión global de los turistas que viajan hacia este destino turístico, y el desarrollo de modelos más complejos que incluyan variables como la satisfacción, el compromiso o el efecto boca-oreja. En este nuevo modelo podríamos incluir la variable satisfacción y el constructo compromiso y serviría para comparar con este primer trabajo las perspectivas que tienen los que no han estado con los que sí han estado en el destino. De esta forma, se podría valorar la diferencia entre las emociones que inspira un destino antes de acudir a él y las emociones activadas por el destino una vez que se está o se ha estado en el destino. Por último, podríamos estudiar el papel moderador de las características y estilos de vida del turista en el modelo global. Así, habrá turistas que no busquen información antes de ir a un destino, simplemente van, y otros que acudan por trabajo en lugar de por vacaciones. Otro ejemplo en este sentido es la diferencia entre turistas psicocéntricos -más introvertidos, menos arriesgados, más conservadores, buscan destinos conocidos- y alocéntricos -más predispuestos a la aventura, más extrovertidos, atraídos por los destinos desconocidos(Plog, 1977), que también puede implicar diferencias en el modelo estudiado.

\section{REFERENCIAS BIBLIOGRÁFICAS}

ACERENZA, M.A. (2003): “Gestión de marketing turístico en el ambiente competitivo actual”, Red de Revistas Científicas de América Latina y el Caribe, España y Portugal, Vol. 2, pp. 43-56. 
ACHROL, R. (1991): "Evolution of the Marketing Organization. New Forms for Turbulent Environments", Journal of Marketing, Vol. 55, No. 4, pp. 77-93.

ANDALEEB, S.S. (1996): "An experimental investigation of satisfaction and commitment in marketing channels: the role of trust and dependence", Journal of Retailing, Vol. 72, No. 1, pp. 77-93.

ANDERSON, E. Y B. WEITZ (1992): "The use of pledges to build and sustain commitment in distribution channels", Journal of Marketing, Vol. 29, pp. 18-34.

BAGOZZI, R.P. (1997): "Goal-directed Behaviours in Marketing: The Role of Emotion, Volition, and Motivation", Psychology and Marketing, Vol.4, No. 3, pp. 309-313.

BAGOZZI, R. P.; GOPINATH, M. Y NYER, P.U. (1999): "The Role of Emotions in Marketing”, Journal of the Academy of Marketing Science, Vol. 27, pp. 184-206.

BAGOZZI, R.P., BAUMGARTNER, H., PIETERS, R. Y ZEELENBERG, M. (2003): "The role of emotions in goal-directed behaviour", capítulo 3 del libro The Why of Consumption. Contemporary perspectives on consumer motives, goals and desires (Eds. Ratneshwar, S., Mick, D.G. y Huffman, C.), Routledge, pp. 36-58.

BART, Y., SHANKAR, V., SULTAN, F., URBAN,G.L. (2005): "Are the drivers and role of on-line trust the same for all web sites and consumers? A large -Scale Exploratory empirical study", Journal of Marketing, Vol. 69, No. 4, pp. 133-153.

BELLMAN, S.; LOHSE, G.L. Y JOHNSON, E.J. (1999): "Predictors of Online Buying Behavior", Communications of the ACM, Vol. 42, No. 12, pp. 32-38.

BENNETT, R. Y GABRIEL H. (2001): "Reputation, trust and supplier commitment: the case of shipping company/seaport relations", Journal of business and Industrial Marketing; Vol. 16, No. 6; pp. 424-438.

BERGEN, M., S. DUTTA Y O.C.WALKER JR. (1992): "Agency relationships in marketing: a review of the implications and applications of agency and related theories", Journal of Marketing, vol. 56, (Julio), pp. 1-24.

CHU, W. Y CHU, W. (1994): "Signalling quality by selling through a reputable retailer: an example of renting the reputation of another agent", Marketing Science, Vol. 13, No. 2, pp. 177-189.

CLARK, M. S. E ISEN, A. M. (1982): "Towards understanding the relationship between feeling states and social behaviour", en A. H. Hastorf y A.M. Isen (eds), Cognitive Social Psychology, New York: Elsevier-North Holland.

DERBAIX, C. Y PHAM, M.T. (1998): "For the Development of Measures of Emotion in Marketing: Summary of Prerequisites", in M. Lambkin, G. Foxall, T. Van Raaij and B. Heilbrum (eds.) European Perspectives on Consumer Behavior, London: Prentice Hall, pp. 140-155.

DONEY, P.M Y CANNON, J.P (1997): "An Examination of the Nature of Trust in Buyer-Seller Relationships", Journal of Marketing, Vol. 61, No. 2 (abril), pp. 35-47.

DUNN, J.R. Y SCHWEITZER, M.E. (2005): "Feeling and believing: The influence of emotion on trust", Journal of Personality and Social Psychology, Vol. 88, N. 5, pp. 736-748

DWYER, F.R., P.H. SCHURR Y S. OH (1987): "Developing buyer-seller relationships", Journal of Marketing, Vol. 51, abril, pp. 11-27.

ERDEM, T. Y J. SWAIT (1998): "Brand equity as a signalling phenomenon", Journal of Consumer Psychology, Vol. 7, No. 2, pp. 131-158.

FARRELLY, F. Y QUESTER, P. (2003): "The effects of market orientation on trust and commitment", European Journal of Marketing, Vol. 37, No. 3/4, pp. 530-553.

FOMBRUN, C. (1998): "Indices of corporate reputation: An analysis of media rankings and social monitors' ratings", Corporate Reputation Review, Vol. 1 No.4, pp.327-40.

FORNELL, C. AND LACKER, D. (1981): "Evaluating structural equation models with unobservable variables and measurement error", Journal of Marketing Research, Vol. 18, pp. 39-50

FRIJDA, N. H. (1986): The emotions, Ed. Cambridge University Press, Cambridge, Reino Unido.

GANESAN, S. (1994): "Determinants of long-term Orientation in Buyer-Seller Relationships", Journal of Marketing, Vol. 58, No. 2 (abril), pp. 1. 
GANESAN, S. Y HESS, R. (1997): "Dimensions and Levels of Trust: Implications for Commitment to a Relationship", Marketing Letters, Vol. 8, No. 4, pp. 439-448.

GARBARINO, E. Y JOHNSON M.S (1999): "The Different Roles of Satisfaction, Trust, and Commitment in customer Relationship", Journal of Marketing, Vol. 63, No. 2 (abril), pp. 70.

GARDNER, MERYL P. (1985): "Mood States and Consumer Behavior: A Critical Review”, Journal of Consumer Research, Vol. 12 (diciembre), pp. 281-300.

GÓBE, M (2001): Emotional Branding: The New paradigm for connecting brands to people. USA, Watson-Guptill Public.

GOOSENS, C. (2000): "Tourism Information and Pleasure Motivation", Annals of Tourism Research, Vol. 27, No. 2, pp. 301-321.

GUMMESSON, E. (1996): "Relationship marketing and imaginary organizations: a synthesis", European Journal of Marketing, Vol. 30, No. 2, pp. 31-44.

GUNDLACH, G.T; ACHROL, R.S Y MENTZER, J.T (1995): "The Structure of Commitment in Exchange", Journal of Marketing, Vol. 59, No. 1 (enero), pp. 78.

HAIR, J.F.; R.E. ANDERSON, R.L. TATHAM Y W.C. BLACK (1999): Análisis multivariante de datos. Ed. Prentice Hall, Madrid.

HERBIG, P. Y MILEWICZ, L. (1995): “The relationship of reputation and credibility to brand success", Journal of Consumer Marketing, Vol. 12, No. 4, pp. 5-10.

HERBIG, P. Y MILEWICZ, J. (1996): "Market signalling: a review", MCB University Press, Vol. 34, No. 1, pp. 35-45.

HESS, J. Y STORY, J (2005): “Trust-Based Commitment: Multidimensional Consumer-Brand Relationships", Journal of Consumer Marketing, Vol. 22, No. 6, pp. 313 - 322.

HUANG, M.H. (2001): "The Theory of Emotions in Marketing", Journal of Business and Psycho$\log y$, Vol. 16, No. 2, pp. 239-247.

IZARD, C.E. (1977): “Human Emotions", Plenum Press, Nueva York.

KIRMANI, A. Y RAO, A.R. (2000): "No pain, no gain: A critical review of the literature on Signaling Unobservable Product Quality”, Journal of Marketing, Vol. 64, No. 2, pp. 66-79.

KREPS, D.V. Y WILSON, R. (1982): "Reputation and imperfect information", Journal of Economic Theory, Vol. 27, pp. 253-279.

LILJANDER, V. Y STRANDVIK, T. (1997): "Emotions in Service Satisfaction", International Journal of Service Industry Management, Vol. 8, No. 2, pp. 148-169.

MACINTOSH, G. Y L.S. LOCKSHIN (1997): "Retail relationship and store loyalty: a multi-level perspective", International Journal of Research in Marketing, Vol. 14, pp. 487-497.

MEMELSDORFF, F. (1998): "Marketing estratégico en turismo: branding, identidad y cultura corporativa”, Revista Valenciana d Estudis Autonómics, Vol. 25, pp. 73-80.

MITCHELL Y VINCENT-WAYNE. (1999): “Consumer Perceived Risk: Conceptualisations and Models", European Journal of Marketing, Vol. 33, No. 1, pp. 163-195.

MOORMAN, C., R. DESHPANDÉ Y G. ZALTMAN (1993): "Factors affecting trust in market research relationships", Journal of Marketing, Vol. 57 (enero), pp. 81-101.

MORGAN, R.M Y HUNT, S.D (1994): “The Commitment -Trust Theory of Relationship Marketing", Journal of Marketing, Vol. 58, No. 3, pp. 20-38.

NOOTEBOOM, B. Y N.G. NOORDERHAVEN (1997): "Effects of trust and governance on relational risk", Academy of Management Journal, Vol. 40, No. 2, pp. 308-338.

OLIVER, R.L. (1997): Satisfaction: A Behavioural Perspective on the Customer, New York: McGraw Hill.

PLUTCHIK, R. (1980): Emotion: A Psychoevolutionary synthesis. New York: Harper y Row.

PLOG, S. (1977): "Why destinations rise and fall in popularity", en Kelly, E. (ed.) Domestic and International Tourism, Wellsbury, MA, Institute of Certified Travel Agencies.

RESNICK J.T. (2003): Reputation: The Inside Story, Pharmaceutical Executive, Vol.1 (Junio) 
RESTALL, C. Y GORDON, W. (1993): "Brands- The missing link; Understanding the emotional relationship", Marketing and Research Today, Vol. 21, No. 2. (mayo) Amsterdam, pp. 59-68.

RICHINS, M.L. (1997): "Measuring Emotions in the Consumption Experience", Journal of Consumer Research, Vol. 24, No. 2, pp. 127-146.

RUSSELL, J.A. (1980): "A Circumplex Model of Affect", Journal of personality and Social Psychology, Vol. 39, pp. 1161-1178.

RUSSELL, J.A., Y MEHRABIAN, A. (1977): "Evidence for a three-factor theory of emotions", Journal of Research in Personality, Vol. 11, pp. 273-294.

SAKO, M Y S. HELPER (1998): "Determinants of trust in supplier relations: evidence from the automotive industry in Japan and the United States", Journal of Economic Behavior and Organization, Vol. 34, pp. 387-417.

SAN MARTÍN, S. (2003): La relación del consumidor con las agencias de viajes, Universidad de Burgos, Burgos.

SELNES, F. (1998): "Antecedents and consequences of trust and satisfaction in buyer-seller relationships", European Journal of Marketing, Vol. 32, No. 3/4, pp. 305-322.

SHAPIRO, C. (1982): "Consumer information, product quality, and seller reputation", The Bell Journal of Economics, Vol. 13, No. 1, pp. 20-35.

SINGH, J. Y D. SIRDESHMUKH (2000): "Agency and trust mechanisms in consumer satisfaction and loyalty judgments", Journal of the Academy of Marketing Science, Vol. 28, No. 1, pp. 150167.

STAUSS, B. Y NEUHAUS, P. (1997): “The qualitative satisfaction model”, International Journal of Service Industry Management, Vol. 8, No. 3, pp. 236-249.

WATSON, D., Y CLARK, L.A. (1992): "Affects separable and inseparable: On the hierarchical arrangement of the negative affects", Journal of Personality and Social Psychology, Vol.62, pp. 489-505.

WETZELS M., DE RUYTER K. Y VAN BIRGELEN M. (1998): "Marketing service relationships: the role of commitment", Journal of Business and Industrial Marketing, Vol. 13, No. 4/5, pp. 406-423.

WONG, A. (2004): "The role of emotional satisfaction in service encounters", Managing Service Quality, Vol. 14, No. 5, pp. 365-376.

ZEITHALML, V.A., LEONARD, L.B. Y PARASURAMAN, A. (1996): “The Behavioral Consequences of Service Quality”, Journal of Marketing, Vol.60 (abril), pp. 31-46. 


\section{APÉNDICE Ítemes de las escalas de medida de las variables de estudio realizado}

\begin{tabular}{|c|c|c|c|c|c|c|c|c|c|c|c|c|c|c|c|c|c|c|c|c|}
\hline 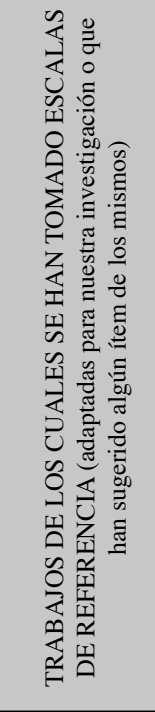 & 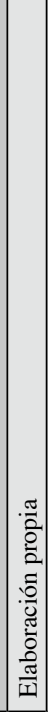 & 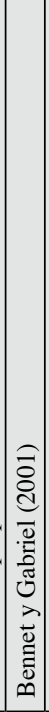 & 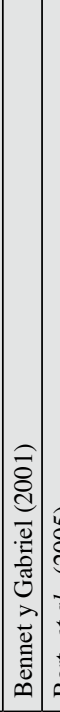 & 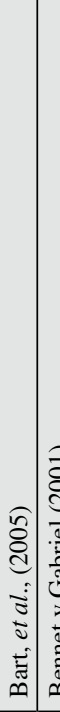 & 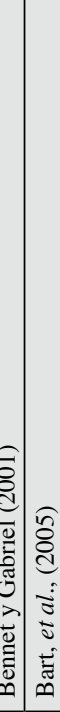 & 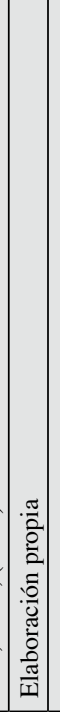 & & 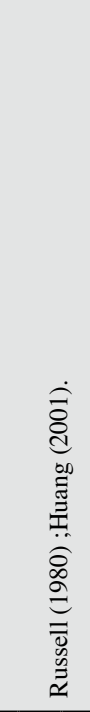 & & & 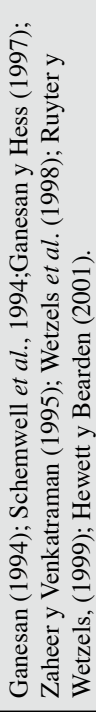 & 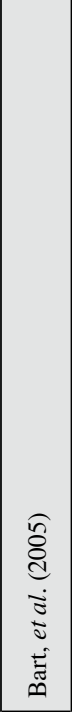 & 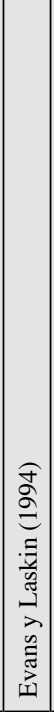 & 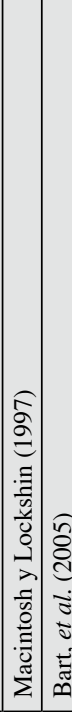 & 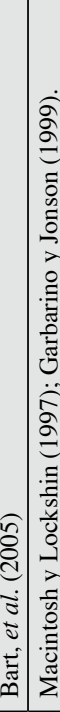 & 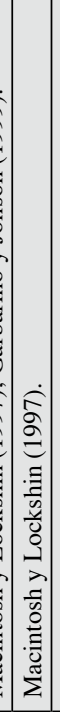 & 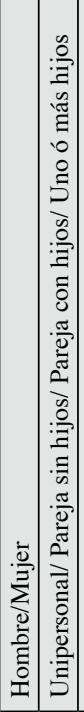 & 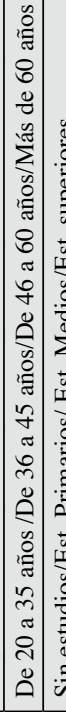 & 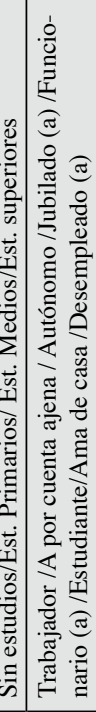 & 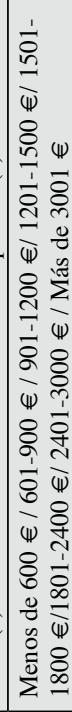 \\
\hline 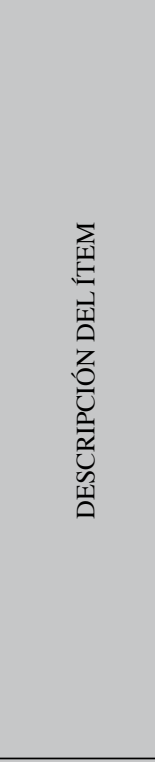 & 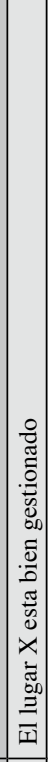 & 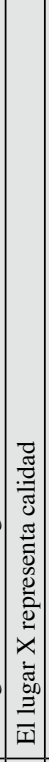 & 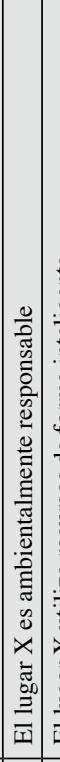 & 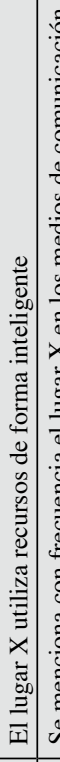 & 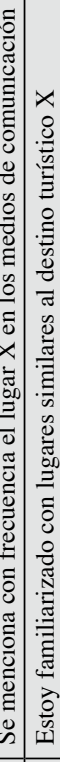 & 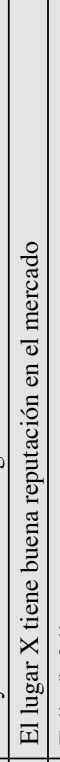 & 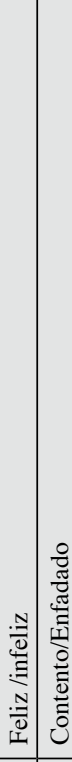 & 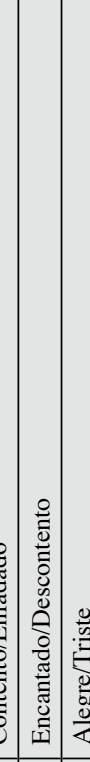 & 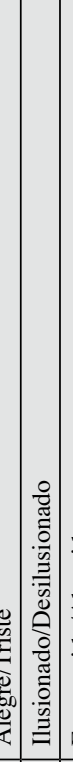 & 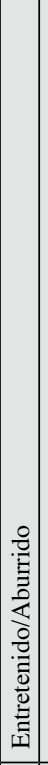 & 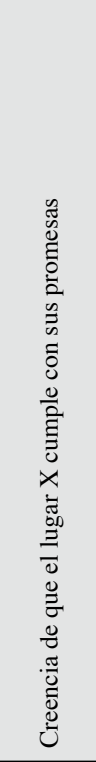 & 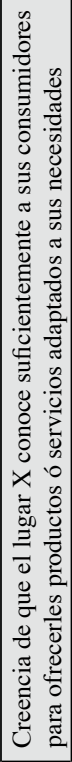 & 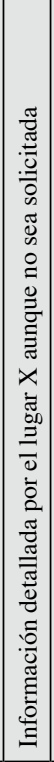 & 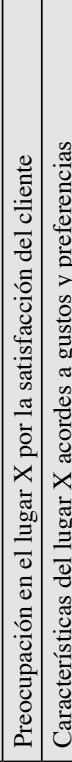 & 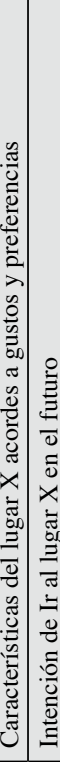 & 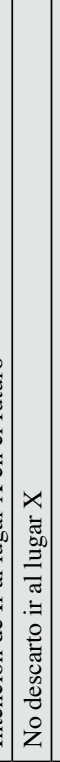 & 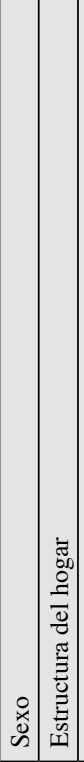 & (2) & 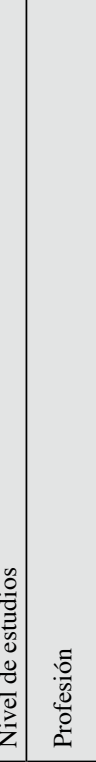 & 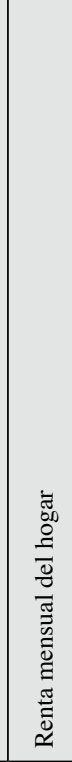 \\
\hline иоџฺрвуц!ро ว & 9 & $\stackrel{0}{>}$ & 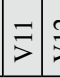 & $\stackrel{\overbrace{}}{>} \stackrel{5}{=}$ & $\stackrel{2}{>}$ & $\stackrel{n}{>}$ & $\stackrel{0}{>} \stackrel{5}{=}$ & $=\stackrel{\infty}{>} \frac{9}{>}$ & $\vec{i}$ & $\vec{s}$ & กิ & $\stackrel{\overbrace{}}{>}$ & $\stackrel{+}{S}$ & 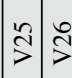 & $\stackrel{\substack{j \\
>}}{>}$ & $\stackrel{\infty}{\stackrel{\infty}{>}}$ & ঙे & $\vec{s}$ & $\begin{array}{c}\stackrel{1}{s} \\
\stackrel{3}{>}\end{array}$ & 4 \\
\hline 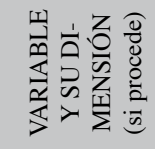 & & \multicolumn{4}{|c|}{ 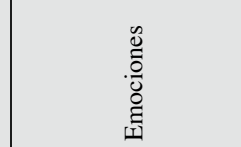 } & \multicolumn{4}{|c|}{ ปี } & & & \multicolumn{4}{|c|}{ 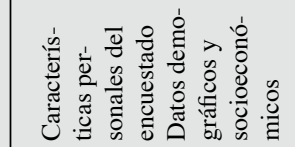 } \\
\hline
\end{tabular}

*Nota: de V1 a V8 se preguntaba el grado de conocimiento de las distintas zonas de Mundo Maya-México, por lo que no son escalas. 


\section{Gipuzkoaren kanpo harremanak, esku onetan.}

Las relaciones externas de Gipuzkoa, en buenas manos.

Les relations externes de Gipuzkoa, en bonnes mains. 